\section{Revista dePolítica Económica y Desarrollo Sostenible}

Centro Internacional de Política Económica para el Desarrollo Sostenible
Revista de Política Económica y Desarrollo Sostenible

EISSN: 2215-4167 • Vol. 5 (2) • Enero-Junio, 2020: 1-24

DOI: https://doi.org/10.15359/peds.5-2.3

URL: http://www.revistas.una.ac.cr/politicaeconomica

Revista electrónica semestral publicada por el Centro Internacional de Política Económica para el Desarrollo Sostenible

Universidad Nacional, Lagunilla, Heredia Apartado 2393-3000

\title{
La igualdad de género en las políticas públicas en el marco de los Objetivos de Desarrollo Sostenible
}

\author{
Gender equality in Public Policies within the framework of the Sustainable \\ Development Goals
}

Evelyn Chen Quesada

(iD) https://orcid.org/0000-0002-5280-3134

evelyn.chen.quesada@una.cr

Carolina Hernández Chaves ${ }^{2}$

(iD) https://orcid.org/0000-0001-8248-6187

Olman Segura Bonilla ${ }^{3}$

(iD) https://orcid.org/0000-0002-5461-1769

Fecha de recibido: 15 de enero, 2020, Fecha de aprobado: 5 de mayo, 2020;

Fecha de publicación: 30 de junio, 2020

\section{Resumen}

Esta es una investigación dirigida a analizar las políticas públicas de Costa Rica generadas después del mandato de la Agenda 2030, formulada en el 2015, que incluye 4 ejemplos de políticas internacionales para verificar el cumplimiento del Objetivo ODS-5 "Igualdad de género" y sus 9 metas. Se utiliza una metodología de carácter naturalista en donde se realiza el estudio de 16 documentos de políticas públicas, comparándolos entre sí y bajo la premisa de cumplir las metas formuladas por Naciones Unidas, del ODS-5. Entre los principales hallazgos se determina que solo una de las 12 políticas analizadas cumple con el $100 \%$ de las metas, lo que implica que un $91.67 \%$ de estas carece de una o más de ellas. La principal conclusión respecto al objetivo del trabajo es que se están dando pasos en la vía correcta respecto a la búsqueda de la igualdad de género, aunque todavía son insuficientes. Se muestran los avances y las falencias. Por lo que se recomienda hacer mayores esfuerzos por formularlas y que incluyan las 9 metas, para que nuestra sociedad se transforme en una más inclusiva, equitativa y se elimine la discriminación por género.

Palabras claves: derechos humanos, desarrollo sostenible, género, igualdad de oportunidades, política pública. 
Revista de Política Económica y Desarrollo Sostenible • EISSN: 2215-4167

Vol. 5 (2) • Enero-Junio, 2020: 1-24

Chen Quesada

DOI: https://doi.org/10.15359/peds.5-2.3

Hernández Chaves

URL: http://www.revistas.una.ac.cr/politicaeconomica

Segura Bonilla

\begin{abstract}
This is an investigation to analyze Costa Rica's public policies generated after the approval of the 2030 Agenda mandate in 2015 and includes 4 examples of international policies to verify compliance with the SDG-5 "Gender Equality" and its nine targets. A naturalistic methodology is used in which the study of 16 public policy documents is carried out, comparing them with each other and under the premise of meeting the United Nations targets of SDG-5. Among the main findings it is determined that only one of the 12 policies analyzed meets $100 \%$ of the targets, implying that $91.67 \%$ of these lack one or more of the 9 targets. The main conclusion regarding the objective of the work is that steps are being taken in the right direction with respect to the search for gender equality, although they are still insufficient. Progress and failures are shown. It is therefore recommended to make greater efforts to formulate public policies that include the 9 goals, so that our society is transformed into more inclusive, equitable and gender discrimination eliminated.
\end{abstract}

Keywords: human rights, gender, equal opportunities, public policy, sustainable development

\title{
Introducción
}

Este estudio es relevante porque identifica las acciones y estrategias gubernamentales para atender los problemas ocasionados por la discriminación de la mujer en los ámbitos social, económico y ambiental, así como la violencia y la desigualdad de género, entre otros. Según la UNESCO (2014), en el Manual Metodológico de Indicadores de Cultura para el Desarrollo ha señalado 7 dimensiones para el desarrollo nacional, la económica, la educación, la gobernanza, la participación social, la igualdad de género, la comunicación y el patrimonio. Este trabajo se centra en el análisis de la Igualdad de Género.

Esta dimensión se basa en la premisa de que la promoción y valorización de la igualdad de género está directamente relacionada con el respeto de la dignidad humana, el disfrute de los talentos que existen en todos los niveles de la sociedad y el aprovechamiento de toda la gama de puntos de vista, obras y desafíos para lograr una vida cultural realmente plena.

El objetivo de esta dimensión es determinar en qué grado la igualdad de género se percibe como algo importante para el desarrollo nacional, el respeto de los derechos humanos (incluidos los derechos culturales) y la construcción de sociedades abiertas e integradoras. (UNESCO, 2014, p.105)

El estudio analiza 16 políticas formuladas, del ámbito nacional e internacional, 12 de Costa Rica, 2 de Guatemala, 1 de Perú y 1 de Colombia, en el marco de la Agenda 2030 y los Objetivos de Desarrollo Sostenible (ODS), aprobada por la Asamblea de las Naciones Unidas en setiembre del 2015. El análisis de estas políticas se realiza según las 9 metas establecidas por el ODS-5 "Igualdad de género". 
Revista de Política Económica y Desarrollo Sostenible • EISSN: 2215-4167

Vol. 5 (2) •Enero-Junio, 2020: 1-24

Chen Quesada

DOI: https://doi.org/10.15359/peds.5-2.3

Hernández Chaves

URL: http://www.revistas.una.ac.cr/politicaeconomica

Segura Bonilla

Sobre la materia, se han realizado investigaciones internacionales acerca de la perspectiva de género, en un estudio sobre los contenidos de sexualidad en los libros de texto de Ciencias Naturales y Formación Cívica y Ética de cuarto, quinto y sexto grados de primaria en los Estados del sureste mexicano. Los resultados muestran que los contenidos de los libros reducen la sexualidad a la actividad reproductiva, con ideas heteronormativas y no incorporan la visión de género. Además, "fallan en brindar herramientas útiles para prevenir el abuso sexual... fracasan en denunciar la desigualdad entre hombres y mujeres con el uso/abuso correspondiente del poder" (Rosales y Salinas, 2017, p. 19).

En otro estudio cualitativo, realizado en Tlaxcala México, sobre las maneras en que el estudiantado, según su condición de género, innova acciones a favor del ambiente y promueve la educación ambiental, mostró que hay diferencias y preferencias entre género para innovar y mitigar los problemas. En específico se muestra que a las mujeres les gusta más cuidar el ambiente que a los hombres (Espejel, Flores y Castillo, 2014, p.35).

Estudios realizados sobre ecodesarrollo desde una perspectiva ecofeminista (Melero y Limón, 2017), han mostrado que el desarrollo y la modernidad han afectado los procesos naturales, sociales, culturales y económicos, por lo que sitúa tanto a hombres como a mujeres en la responsabilidad ciudadana con ética civil que motiven una eco justicia con igualdad de derechos civiles y ambientales. El estudio propone la sustentabilidad y la cooperación internacional con una clara asistencia de la mujer, con responsabilidad política.

El concepto de violencia de género en el Derecho Internacional de los Derechos Humanos ha sido sujeto de análisis sobre la discriminación y la violencia contra las mujeres al no hacer referencia a la categoría género como tal. El estudio analiza lo que se entiende por violencia de género desde el Derecho Internacional de los Derechos Humanos y los principales instrumentos de las mujeres, a saber, la Convención sobre la Eliminación de todas las formas de Discriminación contra la Mujer, la Convención Interamericana para Prevenir, Sancionar y Erradicar la Violencia contra la Mujer, entre otras (Orjuela, 2012).

En el ámbito universitario existen investigaciones que apuntan a la reflexión de la violencia de género en la universidad (Lozano y Bautista, 2015). El estudio analiza un enfoque más amplio y complejo de la violencia a partir de un modelo de dicotómico, asimétrico y jerárquico de los sujetos sociales. Por otro lado, existe otro documento sobre la transversalización de la perspectiva de género (Arias, Bustos y Víquez, 2016) en las carreras y la visibilidad de las mujeres en la docencia de la Facultad de Ciencias Sociales de la Universidad Nacional, Costa Rica. Este plantea que todavía se siguen proyectando roles estereotipados en la docencia, y la profesionalización segregada por sexo. "El material bibliográfico de autoría masculina, sigue predominando en la producción del conocimiento... En cuanto al lenguaje inclusivo, su empleo en los programas es sumamente ligero" (Arias, et al., 2016, p. 143).

Aunado a las investigaciones anteriores, la Universidad Nacional de Costa Rica, realizó un diagnóstico institucional en el 2011 por el Instituto de Estudios de la Mujer, sobre la perspectiva de género y las condiciones laborales de las personas funcionarias académicas para medir las 
Revista de Política Económica y Desarrollo Sostenible • EISSN: 2215-4167

Vol. 5 (2) • Enero-Junio, 2020: 1-24

Chen Quesada

DOI: https://doi.org/10.15359/peds.5-2.3

Hernández Chaves

URL: http://www.revistas.una.ac.cr/politicaeconomica

Segura Bonilla

brechas de inequidad, logrando determinar que la participación de la mujer ha ido en incremento en puestos de directoras, sin embargo, se sigue manteniendo la presencia femenina en los puestos tradicionales (Fernández y Sequeira, 2015).

Según las investigaciones realizadas por el Programa Estado de la Nación, (2019) sobre relaciones laborales y género, se señala:

La desaceleración económica actual encuentra un país que enfrenta retos fundamentales reiterados por este Informe: articular mejor la estructura productiva y el régimen de bienestar, implementar una política de empleo con enfoque territorial y de género, incrementar la calificación de la fuerza de trabajo, regular y proteger las nuevas relaciones laborales que surgen del cambio tecnológico, y adaptar la política social para garantizar una adecuada atención de la transición demográfica y el envejecimiento de la población. Los aportes aquí presentados pretenden apoyar tanto el debate público como la generación de esfuerzos de política para abordar estos desafíos (p. 120).

En sí, el objetivo de la exploración es analizar, en el marco de la Agenda de Desarrollo Sostenible 2030 el ODS-5, Igualdad de Género, el cumplimiento de sus metas en las políticas públicas formuladas posterior a setiembre del 2015 en Costa Rica y algunos países de la región. El propósito de este artículo es ser un referente para futuras investigaciones sobre la igualdad de género en las políticas públicas y brindar información relevante según los resultados del estudio.

\section{Marco teórico}

La Agenda 2030 con los Objetivos de Desarrollo Sostenible (ODS) fue aprobada en setiembre del 2015 por la Asamblea de las Naciones Unidas. Esta Agenda contiene 17 ODS que marcan la hoja de ruta para erradicar la pobreza extrema, reducir la desigualdad en todas sus formas, la igualdad de género, la educación, entre otros.

Frente a estos desafíos el objetivo 5 de los ODS, pretende "lograr la igualdad entre los géneros y empoderar a todas las mujeres y las niñas". En particular interesa para este apartado la meta 5.c la cual dicta, "Aprobar y fortalecer políticas acertadas y leyes aplicables para promover la igualdad de género y el empoderamiento de todas las mujeres y las niñas a todos los niveles" (Naciones Unidas, 2018, p. 33).

Siendo este objetivo 5 y sus 9 metas una gran oportunidad para que los países suscritos tomen las medidas públicas necesarias, por medio de políticas, en todos los sectores con el propósito de disminuir las brechas de desigualdad de género y de esta manera eliminar la violencia contra las mujeres y niñas incluida la explotación sexual y trata, eliminar el matrimonio infantil, la mutilación genital femenina, valorar el trabajo doméstico no remunerado, asegurar que la mujer participe en la vida política, económica y pública, acceso a la salud sexual y reproductiva, acceso a la propiedad, uso de las tecnologías de la información y comunicación (TIC) para el 
empoderamiento de la mujeres y por supuesto fortalecer políticas y leyes que promuevan la igualdad de género (Naciones Unidas, 2018, p. 31-33).

El concepto de género e igualdad de género ha sido incluido en la formulación de políticas públicas, el concepto ha sido tema de discusión en diferentes disciplinas. Interesa para este estudio diferenciar el término género del término sexo (Arango y Corona, 2016):

Sexo: Características biológicas (genéticas, endocrinas y anatómicas) empleadas para agrupar a los seres humanos como miembros ya sea de una población masculina o femenina. Si bien estos conjuntos de características biológicas no son mutuamente excluyentes, en la práctica son utilizados para establecer una diferenciación de los humanos dentro de un sistema binario polarizado. (Organización Panamericana de la Salud - OPS, 2014)

Género: Se refiere al conjunto de papeles y relaciones, actitudes, conductas, valores, relaciones de poder socialmente construidos que se atribuyen a los dos sexos de manera diferencial. En tanto que el sexo biológico es determinado por características genéticas, endócrinas y anatómicas, el género es parte de una identidad aprendida, que cambia con el tiempo y que varía enormemente al interior y entre culturas (Health Canada, 2003, p.19).

Consecuentemente, el género se construye a lo largo de la vida y está influenciado por factores sociales, culturales, psicológicos, económicos, de la época y los lugares, siendo así la UNESCO, (2014) señala que:

Por "género" se entienden las construcciones socioculturales que diferencian y configuran los roles, las percepciones y los estatus de las mujeres y de los hombres en una sociedad. Por "igualdad de género" se entiende la existencia de una igualdad de oportunidades y de derechos entre las mujeres y los hombres en las esferas privada y pública que les brinde y garantice la posibilidad de realizar la vida que deseen. Actualmente, se reconoce a nivel internacional que la igualdad de género es una pieza clave del desarrollo sostenible. (p. 104)

La relevancia de considerar la igualdad de género en las políticas públicas es un medio estratégico para asegurar que los derechos humanos se pueden concretar de forma efectiva. La formulación de políticas sensibles al género no solo tiene matices culturales sino también de desarrollo económico y educativos para los diferentes contextos sociales donde se formulen, dependiendo del entorno, la política se define como "un curso de acción o inacción elegido por las autoridades para hacer frente a un problema determinado o conjunto interrelacionado de problemas" (Arango y Corona, 2016, p. 39).

Las políticas públicas deben, al menos, contemplar tres aspectos: el problema que se quiere atender, los objetivos orientadores del proceso y las políticas o medios por los cuales se va a 
Revista de Política Económica y Desarrollo Sostenible • EISSN: 2215-4167

Vol. 5 (2) • Enero-Junio, 2020: 1-24

Chen Quesada

DOI: https://doi.org/10.15359/peds.5-2.3

Hernández Chaves

URL: http://www.revistas.una.ac.cr/politicaeconomica

Segura Bonilla

ejecutar sus acciones. Identificar, describir y cuantificar el problema de género del país, para lo cual debe estar basado en evidencia, por medio de estudios, diagnósticos, bases de datos y otros que aporten información necesaria para comprender la dinámica del problema. El diagnóstico para la planificación debe contener: "Una caracterización de la naturaleza y magnitud de los problemas que requieran atención; sin perder de vista su inserción en el contexto global en que aparecen las relaciones y determinaciones que dan origen y explican la existencia de tales problemas" (Pichardo, 1993, p. 97).

El segundo aspecto se refiere a los objetivos orientadores que están en función del cómo se va a atender el problema y hacia dónde se quiere ir o que se desea alcanzar.

El tercer aspecto son las políticas o los medios mediante los cuales serán abordados y resueltos los problemas. Según Arango y Corona, (2016),

En algunas políticas, los instrumentos se llaman estrategias. Son simplemente los medios mediante los cuales se alcanzarán los objetivos. Los instrumentos pueden contener una estructura para implementar la política, identificar las fuentes de financiación, y tener una lista de diversos asociados para el desarrollo. (p. 39)

La formulación de políticas públicas en la atención de problemas determina el Curso o línea de acción definido para orientar o alcanzar un fin, que se expresa en directrices, lineamientos, objetivos estratégicos y acciones sobre un tema y la atención o transformación de un problema de interés público. Explicitan la voluntad política traducida en decisiones y apoyo en recursos humanos, técnicos, tecnológicos y financieros y se sustenta en los mandatos, acuerdos o compromisos nacionales e internacionales (MIDEPLAN 2016, p.6).

El desarrollo e implementación de políticas públicas conlleva un ciclo que inicia con: 1 . Definición del problema, 2. Configuración de agenda, 3. Formulación de políticas, 4. Adopción de política/legitimación, 5. Implementación de política y 6. Evaluación de política (Arango y Corona, 2016, p. 40). La participación ciudadana en el ámbito nacional, regional y en territorios permite la concertar los temas prioritarios y se constituyen compromisos de todas las partes.

Por la complejidad social de la desigualdad de género y la necesidad de acciones para solucionar este tema es que el ciclo de políticas públicas debe contemplar el objetivo 5 de la ODS en todos sus componentes y además de la consulta de las principales convenciones, acuerdos y declaraciones internacionales referidas a la temática; tal es el caso de, la Convención Internacional sobre la Concesión de los derechos civiles a la mujer, Convención contra todas las formas de discriminación contra la mujer, Convención Interamericana para prevenir, sancionar y erradicar la violencia contra la mujer (Convención de Belem do Pará), las Metas del Milenio, Declaración Universal de los Derechos Humanos, Declaración Primera Conferencia Mundial de la Mujer, México 1975, Declaración de la Segunda Conferencia Mundial de la Mujer, Copenhague 1980, 
Declaración de la Tercera Conferencia Mundial de la Mujer, Nairobi 1985, Declaración de la Cuarta Conferencia Mundial de la Mujer, Beijing 1995, Estrategia Montevideo para la implementación de la Agenda Regional de Genero en el Marco del Desarrollo Sostenible hacia 2030.

El concepto de desarrollo sostenible es el eje transversal en las políticas que contemplan los 17 ODS. Los países de Centroamérica en el manifiesto la Alianza para el Desarrollo Sostenible (2016) lo conceptualizan como:

un proceso de cambio progresivo en la calidad de vida del ser humano, que lo coloca como centro y sujeto primordial del desarrollo, por medio del crecimiento económico con equidad social y la transformación de los métodos de producción y de los patrones de consumo y que se sustenta en el equilibrio ecológico y el soporte vital de la región. Este proceso implica el respeto a la diversidad étnica y cultural regional, nacional y local, así como el fortalecimiento y la plena participación ciudadana, en convivencia pacífica y en armonía con la naturaleza, sin comprometer y garantizando la calidad de vida de las generaciones futuras (p. 154).

Por otro lado, la Asamblea General de las Naciones Unidas define el desarrollo sostenible "como la satisfacción de las necesidades de la generación presente sin comprometer la capacidad de las generaciones futuras para satisfacer sus propias necesidades".

En síntesis, la correlación entre las metas e indicadores del ODS-5 para atender la igualdad de género por medio de políticas públicas efectivas, incluidas de forma transversal en políticas de los diferentes sectores, es una tarea que no puede estar pendiente ya que la evaluación y seguimiento de estas, permiten que se tomen decisiones gubernamentales a tiempo y orientadas por un lado a eliminar la desigualdad de género y por el otro, al empoderamiento de las generaciones de mujeres y niñas en las dimensiones social, económica y ambiental.

\section{Metodología}

El presente estudio responde a un trabajo por indagación naturalística la cual ofrece métodos que sirven para resolver problemas. Kline, 1980 (citado por Scott, 1990),

definió la investigación naturalística como un método para obtener información descriptiva, asociativa, y a veces lógica de causa y efecto sobre las personas, por medio de la observación y las entrevistas en su ambiente natural, de obtener sus informes acerca de acontecimientos, y de analizar documentos y rastros físicos que describen su historia. (p.105)

Este método se ha venido desarrollando como un mecanismo de estudio de las políticas y el sector administrativo en las últimas décadas, ya que utiliza las técnicas comparativas y 
el estudio documental para establecer procedimientos y codificar la información que se está tratando de obtener y que se ha implementado con mayor fuerza en los procesos de pesquisa de las ciencias sociales.

El objetivo fundamental del método comparativo y el análisis documental es establecer y detallar la generalidad empírica y la verificación de hipótesis. Entre las ventajas que se pueden citar dentro del método comparativo están: 1) comprender fenómenos desconocidos a partir de los conocidos, 2) la posibilidad de explicarlos e interpretarlos, 3) perfilar nuevos conocimientos, 4) destacar lo peculiar de fenómenos conocidos, y 5) sistematizar la información al distinguir las diferencias con fenómenos o casos similares (Chen, Hernández, Muñoz y Segura, 2018, p.109) .

Implementar el método comparativo y análisis documental, como en cualquier investigación científica cualitativa requiere un proceso y una cierta secuencia lógica. Para el presente trabajo se establecieron tres etapas; en la primera se estudia la línea del concepto desarrollo sostenible, igualdad de género, políticas públicas, al utilizar el análisis bibliográfico, mediante una lectura selectiva, comprensiva, estratégica y crítica de libros y artículos científicos, a fin de incluir en el estudio insumos teóricos, conceptos y datos relevantes (Chen et al., 2018, p.109) .

En la segunda etapa, se analizan en detalle las políticas públicas que se han venido desarrollando en el ámbito nacional e internacional, que tienen un vínculo con los 17 Objetivos del Desarrollo Sostenible (ODS) según la Agenda 2030, específicamente el ODS-5 "Igualdad de género". Importante hacer notar que no se trata de un análisis de leyes, sino de políticas. Se seleccionan el 100 \% de las nacionales de Costa Rica aprobadas del año 2016 al 2019.

En la tercera etapa, se sistematizan los resultados producto del análisis, se discuten y se presentan en un cuadro donde se muestra el cumplimiento o no de las políticas públicas según el ODS-5 estipulado en la Agenda 2030. Producto de este análisis comparativo, en la última sección se plantean conclusiones y recomendaciones.

\section{Resultados}

Se presenta la Tabla 1 que resume y clasifica el análisis de las 12 políticas públicas de Costa Rica (2016-2018) según el sector al que pertenece, así como los objetivos y los principios orientadores de cada una, de conformidad con el enfoque igualdad de género. Como se puede apreciar en el Gráfico 1, un 59 \% de las políticas costarricenses corresponden al sector social, mientras que el $33 \%$ al sector ambiental y solo el $8 \%$ al sector económico. 
Revista de Política Económica y Desarrollo Sostenible • EISSN: 2215-4167

Vol. 5 (2) •Enero-Junio, 2020: 1-24

Chen Quesada

DOI: https://doi.org/10.15359/peds.5-2.3

Hernández Chaves

URL: http://www.revistas.una.ac.cr/politicaeconomica

Segura Bonilla

\section{Gráfico1.}

Políticas Públicas Costarricenses según sector, 2016-2018

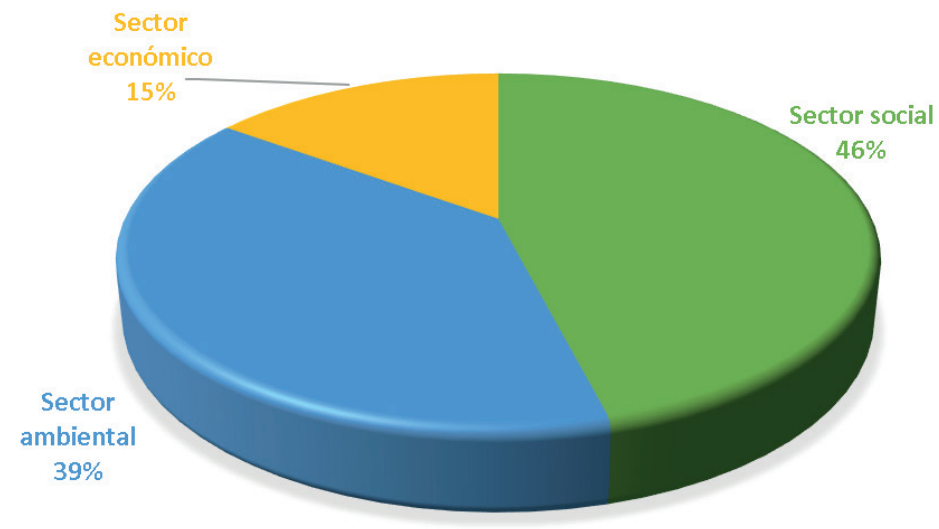

Fuente: Elaboración propia

Tabla 1.

Inclusión del ODS-5 Igualdad de género en las políticas públicas nacionales en Costa Rica

\section{Nombre de la Política}

Sector Social

Política Nacional para la Igualdad Efectiva entre Mujeres y Hombres, (20182030), Instituto Nacional de las Mujeres (INAMU)

Atención y la Prevención de la Violencia contra las Mujeres de todas las Edades, (20172032), Instituto Nacional de las Mujeres (INAMU)

Política Educativa: La persona: centro del proceso educativo y sujeto transformador de la sociedad (2018), Ministerio de Educación Pública

\section{Objetivos}

Cumplir con los compromisos internacionales aprobados por el Estado Costarricense en materia de derechos humanos de las mujeres y el avance hacia la igualdad efectiva entre mujeres y hombres, toma en cuenta las demandas y necesidades de mujeres del país, y apuesta a la reducción de las principales brechas de desigualdad entre mujeres y hombres.

Propiciar percepciones no estereotipadas de las mujeres y los hombres basadas en principios de no violencia, igualdad, relaciones igualitarias, respeto al cuerpo de las mujeres, dignidad y reconocimiento y valoración de las diferencias.

Promover el desarrollo sostenible, estilos de vida saludables y en armonía consigo mismo o consigo misma, las demás personas y el entorno natural; la promoción de una cultura de paz y no violencia, la ciudadanía planetaria y la valoración de la diversidad cultural.

\section{Principios orientadores}

Enfoque Igualdad de género

Comprensión de las necesidades específicas, intereses y oportunidades de las mujeres y los hombres.

El género llama la atención acerca de las construcciones sociales de distinto orden, desde prácticas hasta normas, valores y símbolos asociados a los sexos femenino y masculino.

Promoción de la igualdad sustantiva o de hecho entre sexos, el desarrollo de habilidades, competencias sociales, valores y prácticas de igualdad, respeto a las diferencias, diálogo y solución no violenta de conflictos, la organización y la movilización social.

Articulación de principios clave como la inclusión y la equidad, el respeto a la diversidad, la multiculturalidad y la pluriculturalidad, la sostenibilidad, la resiliencia y la solidaridad, así como las metas educativas que fomentan la formación humana para la vida, con el desarrollo de habilidades, destrezas, competencias, actitudes y valores. 
Revista de Política Económica y Desarrollo Sostenible • EISSN: 2215-4167

Vol. 5 (2) • Enero-Junio, 2020: 1-24

Chen Quesada

DOI: https://doi.org/10.15359/peds.5-2.3

Hernández Chaves

URL: http://www.revistas.una.ac.cr/politicaeconomica

Segura Bonilla

\begin{tabular}{|c|c|c|}
\hline Nombre de la Política & Objetivos & $\begin{array}{c}\text { Principios orientadores } \\
\text { Enfoque Igualdad de género }\end{array}$ \\
\hline $\begin{array}{l}\text { Política Nacional de } \\
\text { Desarrollo Urbano (2018- } \\
\text { 2030) Consejo Nacional de } \\
\text { Planificación Urbana } \\
\text { (Ministerio de Vivienda y } \\
\text { Asentamientos Humanos, } \\
\text { Ministerio de Planificación } \\
\text { y Política Económica, } \\
\text { Ministerio de Obras Públicas } \\
\text { y Transportes... }\end{array}$ & $\begin{array}{l}\text { Integrar un marco de objetivos y esfuerzos } \\
\text { institucionales comunes, que procuren el } \\
\text { desarrollo del territorio y de la población de } \\
\text { manera equilibrada, equitativa, sostenible y } \\
\text { competitiva, mediante la correcta gestión de los } \\
\text { asentamientos humanos y el aprovechamiento } \\
\text { responsable y sostenible de los recursos. }\end{array}$ & $\begin{array}{l}\text { Abolir la discriminación entre ambos } \\
\text { sexos, sin privilegiar injustificadamente } \\
\text { a cualquiera de ellos considerando las } \\
\text { necesidades de la población, niños, } \\
\text { adolescentes, adultos mayores y } \\
\text { personas con alguna discapacidad física, } \\
\text { independientemente del género. }\end{array}$ \\
\hline
\end{tabular}

Ministerio de Ambiente y Energía, Instituto Nacional de Vivienda y Urbanismo, Instituto de Fomento Municipal, Compañía Nacional de Fuerza y Luz, Comisión Nacional de Prevención de Emergencias y el Instituto Nacional de Acueductos y Alcantarillados)

Política Nacional de Responsabilidad Social (20172030) Ministerio de Asuntos Exteriores y de Cooperación

Política Nacional Atención Integral a las Personas en Situación de Abandono y Situación de Calle (2016 2026) Ministerio de Desarrollo Humano e Inclusión Social

Sector Económico
Política Nacional de
Producción y Consumo
Sostenible $(2018-2030)$

Ministerio de Ambiente $y$ Energía

Política Nacional para la igualdad entre mujeres y hombres en la formación, el empleo y el disfrute de los productos de la Ciencia, Tecnología, las Telecomunicaciones y la Innovación 2018-2027
Promover la Responsabilidad Social entre las empresas y organizaciones públicas y privadas para que las buenas prácticas vayan aflorando y generalizándose, a la vez que se involucra y sensibiliza a la población sobre su importancia.

Generar acciones gubernamentales y no gubernamentales articuladas, oportunas, sistemáticas y sostenibles para la prevención, atención y protección de las personas habitantes de calle y personas en riesgo o en situación de abandono.

Promover la adopción de patrones de consumo y producción sostenibles en todos los ámbitos de la economía nacional, que propicien el bienestar social, económico y ambiental de la población en general.

Promover la igualdad en la participación de las mujeres respecto de los hombres en la atracción, la permanencia, la formación, la capacitación, el empleo de calidad y la investigación, en los diferentes campos de la ciencia, la tecnología y la innovación, para facilitar el disfrute de los avances científicostecnológicos.
Promoción en todas las empresas

y organizaciones la igualdad en términos de género que ayuden a la integración de políticas de promoción de la mujer en el ámbito laboral y que, de esa manera, contribuyan a su autonomía económica.

Atención de personas en situación de pobreza extrema y vulnerabilidad mediante la acción articulada. La cobertura de las acciones de esta política incorpora a las personas sin distinción de edad, sexo, género, etnia, condición socioeconómica, discapacidad y nacionalidad.

Participación plena y efectiva de las mujeres y la igualdad de oportunidades de liderazgo a todos los niveles decisorios en la vida política, económica y pública.

Los principios son las orientaciones que guían el accionar de los actores sociales y de las personas comprometidas con esta política. Se expresan en cuatro: la igualdad y la no discriminación, la autonomía y la participación, la universalidad y la articulación. 
Revista de Política Económica y Desarrollo Sostenible • EISSN: 2215-4167

Vol. 5 (2) •Enero-Junio, 2020: 1-24

Chen Quesada

DOI: https://doi.org/10.15359/peds.5-2.3

Hernández Chaves

URL: http://www.revistas.una.ac.cr/politicaeconomica

Segura Bonilla

\begin{tabular}{|c|c|}
\hline Nombre de la Política & Objetivos \\
\hline Sector Ambiental & \\
\hline $\begin{array}{l}\text { Política Nacional de Gestión } \\
\text { del Riesgo (2016-2030) } \\
\text { Comisión Nacional de } \\
\text { Emergencias (CNE) }\end{array}$ & $\begin{array}{l}\text { Promover la gestión del riesgo como concepto } \\
\text { integral del desarrollo, que debe contribuir } \\
\text { al diagnóstico de los factores de riesgo que } \\
\text { subyacen en la actividad social y productiva, en } \\
\text { la adopción de las decisiones y la planificación } \\
\text { en los distintos niveles del territorio y en todos } \\
\text { los sectores, destinada a generar cambios tanto } \\
\text { en la dimensión de los factores físicos como } \\
\text { sociales, causantes del riesgo, para reducir las } \\
\text { pérdidas generadas por desastre y mejorar el } \\
\text { bienestar de las personas. }\end{array}$ \\
\hline
\end{tabular}

Saneamiento en Aguas Lograr, para el año 2045, el manejo seguro del Residuales (2016-2045) total de las aguas residuales generadas en el Instituto Costarricense de país, manejo seguro definido como la garantía Acueductos y Alcantarillados

Ministerio de Salud Ministerio de Ambiente y Energía

Política de Adaptación al Transitarhacia un modelo de desarrollo Cambio Climático (2018- resiliente de la sociedad costarricense, que evite 2030)

Ministerio de Ambiente y del cambio climático.

Energía

Comisión Nacional de

Emergencias

Política Nacional de Agua Procurar el acceso al agua potable por medio Potable de Costa Rica (2017- de la protección del recurso hídrico y el 2030)

Ministerio de Ambiente $y$ Energía

Acueductos y Alcantarillados

Ministerio de Salud

Política Nacional

Humedales (2017-2030)

de

Ministerio de

Ambiente y Energía las pérdidas humanas y moderar los daños materiales generados por los efectos adversos fortalecimiento de las capacidades de los actores relacionados con la prestación del

de que las aguas residuales no afecten al medio ambiente ni a la salud, mediante sistemas de tratamiento individuales o colectivos.

servicio, para contribuir a la salud, bienestar y desarrollo del país.

Gestionar integralmente los ecosistemas de humedal de Costa Rica con el fin de contribuir al desarrollo nacional mediante la conservación de su integridad ecológica y uso sostenible de los servicios ecosistémicos que estos proveen para las actuales y futuras generaciones, haciendo especial énfasis en aquellas poblaciones con dependencia directa de estos ecosistemas.
Incorporación en todas las políticas, planes y procesos de decisión sobre la gestión de los riesgos de desastre, incluidos los relativos a la evaluación de los riesgos, la alerta temprana, la gestión de la información y la educación y la formación.

Acceso a las necesidades de las mujeres y las niñas y las personas en situaciones de vulnerabilidad. a servicios de saneamiento e higiene adecuados y equitativos.

Considera la interrelación entre las diversas vulnerabilidades (social, económica, ambiental) que afectan a las mujeres individual y colectivamente, en particular a las que sufren alguna discapacidad, o son adultas mayores, niñas o adolescentes, entre otros grupos.

Equidad en el acceso al agua potable en todo el territorio nacional mediante la distribución de información, conocimientos, recursos, bienes y servicios, para promover, mejorar y mantener la salud y el desarrollo social en la gestión integral del recurso hídrico.

Aborda el desarrollo sostenible desde la dimensión social, económica y ambiental enfatizando en la equidad. Hace referencia al balance entre la integración de la conservación y el uso sostenible de la diversidad biológica, la cual es crítica para las poblaciones asociadas, tanto por su valor intrínseco para el ecosistema como por el papel que juega en el mantenimiento de los bienes y servicios asociados a su salud, productividad y la sostenibilidad en su calidad de vida y su hábitat.

Fuente: Elaboración propia con base en las 13 Políticas Públicas Nacionales en Costa Rica mencionadas en la primera columna. 
Revista de Política Económica y Desarrollo Sostenible • EISSN: 2215-4167

Vol. 5 (2) • Enero-Junio, 2020: 1-24

Chen Quesada

DOI: https://doi.org/10.15359/peds.5-2.3

Hernández Chaves

URL: http://www.revistas.una.ac.cr/politicaeconomica

Segura Bonilla

En la tabla 2 se presenta la descripción detallada del significado de cada una de las 9 metas con las que se evalúan esas políticas y que han sido establecidas por Naciones Unidas (2018). Posteriormente, en la tabla 3 se presenta una descripción detallada del análisis realizado, el cual, con carácter ilustrativo del conjunto, en un cuadro de agrupamiento presenta una mejor visibilidad de las leyes analizadas, identificando cuáles cumplen y cuáles no, con cada una de las metas de los ODS-5, de acuerdo con la Agenda 2030.

Tabla 2.

Metas del Objetivo de Desarrollo Sostenible 5: Igualdad de género

Metas

5.1 Poner fin a todas las formas de discriminación contra todas las mujeres y las niñas en todo el mundo.

5.2 Eliminar todas las formas de violencia contra todas las mujeres y las niñas en los ámbitos público y privado, incluidas la trata y la explotación sexual y otros tipos de explotación.

5.3 Eliminar todas las prácticas nocivas, como el matrimonio infantil, precoz y forzado y la mutilación genital femenina.

5.4 Reconocer y valorar los cuidados y el trabajo doméstico no remunerados mediante servicios públicos, infraestructuras y políticas de protección social, y promoviendo la responsabilidad compartida en el hogar y la familia, según proceda en cada país.

5.5 Asegurar la participación plena y efectiva de las mujeres y la igualdad de oportunidades de liderazgo a todos los niveles decisorios en la vida política, económica y pública.

5.6 Asegurar el acceso universal a la salud sexual y reproductiva y los derechos reproductivos según lo acordado de conformidad con el Programa de Acción de la Conferencia Internacional sobre la Población y el Desarrollo, la Plataforma de Acción de Beijing y los documentos finales de sus conferencias de examen.

5.a Emprender reformas que otorguen a las mujeres igualdad de derechos a los recursos económicos, así como acceso a la propiedad y al control de la tierra y otros tipos de bienes, los servicios financieros, la herencia y los recursos naturales, de conformidad con las leyes nacionales.

5.b Mejorar el uso de la tecnología instrumental, en particular la tecnología de la información y las comunicaciones, para promover el empoderamiento de las mujeres.

5.c Aprobar y fortalecer políticas acertadas y leyes aplicables para promover la igualdad de género y el empoderamiento de todas las mujeres y las niñas a todos los niveles.

Fuente: Naciones Unidas, 2018, p.31-33

Las interrelaciones de las 9 metas del ODS-5: Igualdad de género, con respecto a las 13 Políticas Públicas Costarricenses objeto de esta investigación, se presentan en la tabla 3. En la primera columna se incluyen los nombres de las trece políticas, las 6 primeras líneas son del sector social, la sétima y octava son del sector económico y las últimas 5 líneas son del sector ambiental. Las nueve columnas siguientes de la tabla son una para cada meta de la 5.1 a la 5.6 y las 5.a, 5.b y 5.c conforme la tabla 2. El cuadriculado que se forma al interceptar las líneas de cada política con las columnas de las metas, nos permite marcar el cumplimiento de estas, de forma tal que es fácil observar cuáles metas del objetivo 5 no se están incluyendo en cada una de las políticas. 
Revista de Política Económica y Desarrollo Sostenible • EISSN: 2215-4167

Vol. 5 (2) •Enero-Junio, 2020: 1-24

Chen Quesada

DOI: https://doi.org/10.15359/peds.5-2.3

Hernández Chaves

URL: http://www.revistas.una.ac.cr/politicaeconomica

Segura Bonilla

La agrupación en este formato permite también visualizar la tendencia que está siguiendo en cada una de las metas, cuáles se están quedando al descubierto y cuáles han sido más fácil de incluir. Igualmente se puede dar mayor importancia al análisis y comprensión de las interrelaciones entre los sectores social, económico y ambiental, cómo se comporta cada uno y cómo se comparan entre ellos respecto a la igualdad de género. Por otro lado, la tabla 3 presenta dos análisis, uno horizontal referido al cumplimiento de las metas de cada una de las políticas aprobadas y otro vertical que permite la comparación de las metas contenidas en el conjunto de las 13 políticas analizadas.

\section{Tabla 3}

Interrelaciones de las metas del ODS-5: Igualdad de género respecto a las políticas públicas costarricenses

\section{POLÍTICAS PÚBLICAS}

1. Igualdad Efectiva entre Mujeres y Hombres (2018-2030)

2. Atención y Prevención de la Violencia contra las Mujeres de todas las Edades (2017-2032)

3. La persona: centro del proceso educativo y sujeto transformador de la sociedad (2017)

4. Desarrollo Urbano (2018-2030)

5. Responsabilidad Social (2017-2030)

6. Atención Integral a las Personas en Situación de Abandono y Situación de Calle (2016 2026)

7. Política Nacional de Producción y Consumo Sostenible (2018-2030)

8. Para la igualdad entre mujeres y hombres en la formación, el empleo y el disfrute de los productos de la Ciencia, Tecnología, las Telecomunicaciones y la Innovación.

9. Gestión del Riesgo (2016-2030)

10. Saneamiento en Aguas Residuales (2016-2045)

11. Adaptación al Cambio Climático (2018-2030)

12. Agua Potable de Costa Rica (2017-2030)

13. Política de Humedales (2017-2030)

Fuente: Elaboración propia con información extraída de las Políticas Públicas 2016-2018. 
Revista de Política Económica y Desarrollo Sostenible • EISSN: 2215-4167

Vol. 5 (2) • Enero-Junio, 2020: 1-24

Chen Quesada

DOI: https://doi.org/10.15359/peds.5-2.3

Hernández Chaves

URL: http://www.revistas.una.ac.cr/politicaeconomica

Segura Bonilla

De acuerdo con el análisis horizontal de la tabla 3, de las Políticas Nacionales de Costa Rica, se comprobó que únicamente la Política Igualdad Efectiva entre Mujeres y Hombres (1) cumple las 9 metas establecidas para el ODS-5, o sea el 100\% de las mismas. Así, el compromiso con la igualdad de género, los derechos y el empoderamiento de las mujeres es transversal en toda esa política. La política para la igualdad entre mujeres y hombres en la formación, el empleo y el disfrute de los productos de la Ciencia, Tecnología, las Telecomunicaciones y la Innovación (8) cumple 8 de las 9 metas.

Las políticas de Atención y Prevención de la Violencia contra las mujeres de todas las edades (2) y La persona: centro del proceso educativo y sujeto transformador de la sociedad (3) alcanzan solamente 7 de las 9 metas, o sea un 78\% de éstas; mientras que Desarrollo Urbano (4) y Responsabilidad Social (5) llegan a un 56\% de cumplimiento de las metas. El resto de las políticas de Atención Integral a las personas en situación de abandono y situación de calle (6), Producción y consumo sostenible (7), Gestión del riesgo (9), Saneamiento en Aguas Residuales (10), Adaptación al Cambio Climático (11), Agua Potable de Costa Rica (12) y Política de Humedales (13) alcanzan únicamente un 44\% de las metas del ODS-5.

En cuanto al análisis vertical, se observa que en el 100\% de las políticas existe un compromiso de cumplimiento de las metas 5.1 y 5.c. La meta 5.1 se refiere a poner fin a todas las formas de discriminación contra todas las mujeres y las niñas en todo el mundo y la 5.c se refiere a la aprobación y fortalecimiento de políticas acertadas y leyes aplicables para promover la igualdad de género y el empoderamiento de todas las mujeres y las niñas a todos los niveles. Este es el resultado de la voluntad política existente a partir de la aprobación de la Agenda 2030 en 2015, en la cual Costa Rica asumió de forma voluntaria la responsabilidad como país miembro de la UNESCO de incluir en sus políticas los Objetivos de Desarrollo Sostenible. Además, en el 2016, Costa Rica se convirtió en el primer país del mundo en suscribir un Pacto Social por la Implementación y Cumplimiento de los Objetivos de Desarrollo Sostenible. Este Pacto es un compromiso país y promueve acciones de largo plazo para lograr cambios. La inclusión de estas metas es también producto de los lineamientos de MIDEPLAN (2016), de orientar los procesos de planificación indicando que en la construcción de las políticas públicas:

Los enfoques también se integran para potencializar algunas condiciones que sirven para educar o enseñar, experimentar e implementar un punto específico a tratar, de manera preconcebida para promover la equidad. Entre los enfoques de las políticas se integra el de Igualdad y Equidad de Género, el enfoque de Derechos Humanos, el Desarrollo Inclusivo, la Interculturalidad, el Enfoque de Desarrollo Sostenible, entre otros (p. 22).

En un $92 \%$ de las políticas aprobadas durante el periodo en estudio, se visualiza la inclusión de la meta 5.5, la cual promueve: "la importancia plena y efectiva de las mujeres y la igualdad de oportunidades de liderazgo a todos los niveles decisorios de la vida política, económica y pública" (Naciones Unidas, 2018, p. 33). El cumplir con esta meta es una oportunidad para 
Revista de Política Económica y Desarrollo Sostenible • EISSN: 2215-4167

Vol. 5 (2) •Enero-Junio, 2020: 1-24

Chen Quesada

DOI: https://doi.org/10.15359/peds.5-2.3

Hernández Chaves

URL: http://www.revistas.una.ac.cr/politicaeconomica

Segura Bonilla

renovar el compromiso por una inclusión efectiva de las mujeres en todos los ámbitos. De acuerdo con Benavente y Valdés, (2014) respecto a la autonomía en la toma de decisiones y la participación femenina se plantea que:

las buenas prácticas de políticas públicas para una democracia inclusiva son las que reconocen a las mujeres como sujetas políticas y establecen medidas o acciones para promover la participación en el ámbito de lo público, lo político y particularmente en la toma de decisiones, como leyes de cuotas, de paridad y de alternancia, y acciones afirmativas, entre otras. (p.12)

En oposición a las metas anteriores que tienen un alto porcentaje de cumplimiento, la meta 5.4 sobre la valorización del trabajo doméstico no remunerado y la responsabilidad compartida de la pareja en el hogar, se invisibiliza en 11 de las 13 políticas estudiadas, o sea un $85 \%$ de las políticas.

Según INAMU (s.f.), citando al Instituto Nacional de Estadísticas y Censos (INEC), en Costa Rica existen 614.025 personas que no participan en actividades económicas por "obligaciones del propio hogar"; de este total-país, 569.664 son mujeres y 44.361 son hombres. (párr. 2). Esta práctica continúa siendo, de acuerdo con el INAMU (s.f.) un hecho socialmente construido, que asigna espacios, roles y prácticas para las mujeres y los hombres, creando así, una separación artificial entre el mundo de lo femenino y el mundo de lo masculino. (parr.3). Sin embargo, empieza a haber consciencia de la situación y se plantea revertir el proceso mediante el cumplimiento de esta meta y de la Ley Contabilización del aporte del trabajo doméstico no remunerado en Costa Rica.

Contrariamente, con miras a enfrentar la Cuarta Revolución Industrial y a experimentar un acelerado proceso de innovación tecnológica que altera la forma en la que vivimos, trabajamos, y nos relacionamos con los demás, y quienes no se adapten a estos cambios quedarán rezagados. Apenas un $38 \%$ de las políticas fomentan la meta 5.b sobre el uso de las TICs para empoderar y promover a las mujeres. En este caso es necesario no solo replantearse la reducción de las brechas de acceso y el uso de las tecnologías; sino también, avanzar hacia el desarrollo de las tecnologías en el marco de un nuevo paradigma productivo y tecnológico, estimulando el interés de las niñas por las ciencias, tecnologías, ingenierías y matemáticas (STEM por sus siglas en inglés), tal y como lo plantea la política por la igualdad de mujeres y hombres en el interés por la Ciencia, Tecnología, las Telecomunicaciones y la Innovación (8).

En el $85 \%$ de las políticas (11 de 13) se omite la meta 5.3, relacionada con la eliminación de prácticas nocivas, como el matrimonio infantil, precoz y forzado. Pese a que la educación en Costa Rica tiene cobertura en todo el territorio nacional y es el medio por excelencia para hacer frente a los factores sistémicos y subyacentes que permiten la existencia del matrimonio infantil, la Política Educativa no la considera explícitamente. Esta omisión es incongruente porque todavía se considera el matrimonio como una forma de asegurar la subsistencia económica de las niñas y las mujeres que no tienen acceso por sí mismas a recursos productivos y viven 
Revista de Política Económica y Desarrollo Sostenible • EISSN: 2215-4167

Vol. 5 (2) • Enero-Junio, 2020: 1-24

Chen Quesada

DOI: https://doi.org/10.15359/peds.5-2.3

Hernández Chaves

URL: http://www.revistas.una.ac.cr/politicaeconomica

Segura Bonilla

en situaciones de extrema pobreza. La anterior práctica lamentable se evidenció en el Censo Nacional realizado en el 2011, el cual señaló que alrededor de 10.321 niñas y adolescentes entre 12 y 17 años, reportaron estar en una relación de convivencia (matrimonio o unión libre). A su vez, el documento Estadísticas Vitales 2016: población, nacimientos, defunciones y matrimonios, realizado por el INEC, visualiza este aspecto e indica que de 26.718 matrimonios reportados, 1.289 son mujeres con edades entre los 15 a 19 años. Vale apuntar que en Costa Rica se aprobó en 2017 la Ley 9406 conocida como Ley de Relaciones Impropias; sin embargo, esta prohíbe las relaciones entre menores de 13 y 15 años y adultos que sobrepasen a estos en 5 o más años.

En el 85 \% de las políticas (11 de ellas), se observa la inclusión de la meta 5.a, que establece la igualdad de derechos de las mujeres a los recursos económicos, ambientales y sociales. Esta meta está regulada por marcos jurídicos y normativos que proporcionan una base para el desarrollo de políticas destinadas a garantizar la igualdad de oportunidades y el acceso a derechos y recursos.

Otro aspectos a destacar es que solo en el $42 \%$ de las políticas se incluye la meta 5.2, se referida a eliminar todas las formas de violencia contra todas las mujeres y las niñas en los ámbitos público y privado. Las políticas que la incluyen son las del sector social, las seis primeras y una del sector económico, la de igualdad de género para el disfrute de la Ciencia, Tecnología, Telecomunicaciones e Innovación, dejando por fuera una del sector económico y todas las del sector ambiental. Sobre esta situación, las cifras en el país son alarmantes según datos del Observatorio de violencia de género contra las mujeres y acceso a la justicia, (Poder Judicial de Costa Rica, 2019) se indica que en el 2018 se contabilizaron 26 femicidios de 64 homicidios contra mujeres.

A nivel nacional, para el mismo año, los delitos de ámbito sexual representaron el 5.9\% de la totalidad de las denuncias interpuestas ante el Ministerio Público contra personas mayores de edad, siendo la cuarta causa de delito por título del Código Penal ingresados como nuevos cada año. Entre el $1^{\circ}$ de enero de 2010 y el 31 de diciembre de 2018, fueron solicitadas un total de 433.483 medidas de protección, para un promedio de 132 medidas de protección por día. En la mayoría de los casos, son mujeres las que solicitan la protección en contra de hombres (pareja sentimental, cónyuge, hermano, padre, tío, abuelo, novio, primo, entre otros). De lo anterior, se colige la importancia de replantear e incluir explícitamente esta meta en todas las políticas públicas.

Con respecto a la meta 5.6, asegurar el acceso universal a la salud sexual y reproductiva y los derechos reproductivos, solo un $38 \%$ de las políticas la incluyen. De nuevo, las políticas sociales son las que incluyen el tema y una de las económicas, el resto no lo consideran. Esto significa que todavía hay tabúes relacionados con la sexualidad y por ende las mujeres no cuentan con las condiciones de equidad necesarias para tomar decisiones de manera voluntaria e informada. Según la Comisión Interamericana de Derechos Humanos (2011):

El derecho de las mujeres de acceso a la información en materia reproductiva hace surgir una obligación proactiva u oficiosa a cargo del Estado, debido al reconocimiento de las limitaciones que suelen tener las mujeres, particularmente las mujeres pobres, indígenas 
Revista de Política Económica y Desarrollo Sostenible • EISSN: 2215-4167

Vol. 5 (2) •Enero-Junio, 2020: 1-24

Chen Quesada

DOI: https://doi.org/10.15359/peds.5-2.3

Hernández Chaves

URL: http://www.revistas.una.ac.cr/politicaeconomica

Segura Bonilla

y/o afrodescendientes y quienes habitan en zonas rurales, para acceder a información confiable, completa, oportuna y accesible que les permita ejercer sus derechos o satisfacer sus necesidades (p.10).

\section{Discusión: sobre lo que realmente sucede con el cumplimiento de metas del ODS-5 en las políticas en Costa Rica}

Un análisis parcial de las políticas de género, por ejemplo, al revisar la inclusión de las 9 metas del objetivo 5 de los ODS en la política de Igualdad Efectiva entre Mujeres y Hombres (2018-2030), podría llevar a concluir que el avance es altamente positivo, al comprobarse que todas las metas de Naciones Unidas fueron tomadas en cuenta en esa política. Sin embargo, este trabajo realiza un análisis mucho más profundo, considerando el conjunto de las 13 políticas públicas emitidas en Costa Rica desde que se aprobaron los ODS en el 2015. Se demuestra que el escenario es más complejo y el cumplimiento de las metas no es como a primera vista se percibe.

Se comprueba en esta investigación que en 11 de las 13 políticas nacionales estudiadas, no se incluyó la meta 5.4 referida a "reconocer y valorar los cuidados y el trabajo doméstico no remunerados mediante servicios públicos, infraestructuras y políticas de protección social, y promoviendo la responsabilidad compartida en el hogar y la familia, según proceda en cada país"; lo mismo sucede con la inclusión de la meta 5.3 referida a eliminar todas las prácticas nocivas, como el matrimonio infantil, precoz y forzado y la mutilación genital femenina. Este es un aspecto sumamente importante para las metas laboral y económica del desarrollo nacional y la omisión se puede calificar como una falta grave, pues el reconocimiento y valoración del trabajo doméstico se encuentra entre las prioridades del país; y por otro lado, el matrimonio infantil, precoz y forzado refuerza la desigualdad $\mathrm{y}$ las relaciones de poder entre el hombre y la mujer y se consideran impropias.

Otros de los incumplimientos mayores de las metas ODS-5 en las políticas analizadas, es lo referido a la sexualidad femenina. En concreto las metas 5.2 respecto a "eliminar todas las formas de violencia contra todas las mujeres y las niñas en los ámbitos público y privado, incluidas la trata y la explotación sexual y otros tipos de explotación", la 5.3 sobre "eliminar todas las prácticas nocivas, como el matrimonio infantil, precoz y forzado y la mutilación genital femenina" y la 5.6 sobre "asegurar el acceso universal a la salud sexual y reproductiva y los derechos reproductivos según lo acordado de conformidad con el Programa de Acción de la Conferencia Internacional sobre la Población y el Desarrollo, la Plataforma de Acción de Beijing y los documentos finales de sus conferencias de examen", no fueron incluidas en la mayoría de las políticas analizadas. Para el caso de la meta 5.2 solamente 6 documentos de políticas la consideran; 7 no lo hacen. La meta 5.3 tiene 2 políticas que sí cumplen, y 11 restantes que no lo hacen. Al final, solo en 5 políticas se pudo observar que se incluye la meta 5.6, lo que señala que 8 políticas de diferentes sectores no lo hacen. Las metas políticas referidas a la sexualidad son también claves para cambiar la actitud machista mayormente generalizada en la sociedad. 
Revista de Política Económica y Desarrollo Sostenible • EISSN: 2215-4167

Vol. 5 (2) • Enero-Junio, 2020: 1-24

Chen Quesada

DOI: https://doi.org/10.15359/peds.5-2.3

Hernández Chaves

URL: http://www.revistas.una.ac.cr/politicaeconomica

Segura Bonilla

Estos hallazgos llevan a concluir que si bien es cierto Costa Rica ha avanzado un poco en la concienciación de la igualdad de género, los desarrolladores de políticas públicas deben profundizar en el análisis e incluir las 9 metas señaladas por Naciones Unidas. De esta forma se puede contribuir al logro del objetivo de igualdad de género de manera transversal en todas las políticas que se generen y se evitarían sesgos, donde las mujeres se visualizan como titulares de derechos y no como medios efectivos para mejorar la productividad, el crecimiento económico y la movilidad social.

En síntesis, se cumple con el objetivo de esta investigación de analizar las políticas públicas aprobadas en Costa Rica después de la aprobación de los Objetivos para el Desarrollo Sostenible por Naciones Unidas en el 2015 y se comprueba que cuando se realiza un análisis integral de las políticas públicas aprobadas (2016-2019), la inclusión de todas las metas del ODS-5 es bastante limitada e incompleta. Solamente una de las trece políticas estudiadas de Costa Rica cumple con todas las 9 metas establecidas.

\section{Conclusiones y recomendaciones}

Este estudio analiza de manera integral y sistémica un tema de actualidad como lo es el cumplimiento del Objetivo 5 de igualdad de género, de los Objetivos del Desarrollo Sostenible (ODS). Algunas personas confunden el objetivo 5 de los ODS como independiente, aislado, referido con gran peso a la necesidad de alcanzar la igualdad de género, pero como si fuera un tema desconectado del resto de las políticas públicas, y no lo es. Aquí se estudia como elemento fundamental que forma parte de un sistema de relaciones y valores, vinculado con el entorno y que, de acuerdo con la Agenda 2030, debe estar presente en todos los esfuerzos por alcanzar el desarrollo.

Se verifica el escaso cumplimiento de las 9 metas establecidas por las Naciones Unidas para el cumplimiento del ODS-5 en 13 políticas públicas aprobadas en Costa Rica entre los años 2016 y 2019. Entre los hallazgos más relevantes se encuentra que solo una política incluye todas las metas, esta es la de Igualdad Efectiva entre Mujeres y Hombres (2018-2030). A la vez, existen algunas políticas nacionales que se quedan muy cortas con el cumplimiento de orientar las condiciones para la igualdad de género. En concreto, 6 de las 13 políticas estudiadas, cumplen con 4 de las 9 metas del ODS-5, o sea alcanzan un 46\% de ellas. Estas políticas son: la de Atención Integral a las Personas en Situación de Abandono y Situación de Calle (6), Producción y Consumo Sostenible (7), Gestión del Riesgo (9), Saneamiento en Aguas Residuales (10), Adaptación al Cambio Climático (11), Agua Potable de Costa Rica (12) y Humedales (13). La anterior situación evidencia y ratifica que persisten importantes obstáculos culturales, sociales y económicos que impiden el cumplimiento de las obligaciones establecidas en los ODS entre otros acuerdos internacionales.

Las dos metas que menor inclusión tienen en las políticas públicas estudiadas son la meta 5.3 y 5.4. La meta 5.3 considera "Eliminar todas las prácticas nocivas, como el matrimonio infantil, precoz y forzado y la mutilación genital femenina”, lo cual es preocupante para el país dada la alta tasa de nacimientos cuyas madres son menores de edad y la cantidad de matrimonios 
Revista de Política Económica y Desarrollo Sostenible • EISSN: 2215-4167

Vol. 5 (2) •Enero-Junio, 2020: 1-24

Chen Quesada

DOI: https://doi.org/10.15359/peds.5-2.3

Hernández Chaves

URL: http://www.revistas.una.ac.cr/politicaeconomica

Segura Bonilla

entre hombres y mujeres menores de 18 años. La meta 5.4 considera "reconocer y valorar los cuidados y el trabajo doméstico no remunerados mediante servicios públicos, infraestructuras y políticas de protección social, y promoviendo la responsabilidad compartida en el hogar y la familia, según proceda en cada país", que en apariencia sería fácilmente atendible, pero que siglos de cultura machista han institucionalizado el tema de los roles del trabajo hombre y mujer.

El estudio también ilustra como Costa Rica mantiene un discurso y una posición fuerte a nivel internacional a favor de la igualdad de género y la aprobación de los ODS que lo incluyen; pero, al mismo tiempo, a lo interno mantiene un gran rezago respecto a dicha igualdad real. Las grandes cantidades de casos de agresión contra la mujer, femicidios y cantidad de matrimonios con niñas y jóvenes menores de edad registrados oficialmente por el INEC dan fe del problema, pero además, el análisis cualitativo realizado en esta investigación muestra como las políticas públicas aprobadas en estos años, no incluyen de forma clara y contundente una posición más enérgica en favor de la igualdad de género demuestra que existen grandes espacios de mejora en esta materia.

Las políticas públicas que consideran más metas del objetivo de igualdad de género son las clasificadas como del sector social y una del sector económico, no las del sector ambiental. Esto indica la carencia de consideración de los objetivos de la Agenda 2030 en los actores que impulsan las políticas ambientales y económicas. Cada vez más las mujeres participan activamente en todas las actividades de los sectores productivos y se considera que hemos ingresado a la Cuarta Revolución Industrial, se hace mucho más relevante para el presente y para el futuro la igualdad de género. No es posible dejar al $50 \%$ de la población, a las mujeres, fuera del nuevo contexto mundial de cambio e innovación tecnológica.

Ante estos hallazgos es sumamente importante realizar esfuerzos a nivel país para transformar la institucionalidad dominante que ha impedido a Costa Rica avanzar en el cumplimiento de las metas del ODS-5 de igualdad de género. Debe transformarse el sentimiento subyacente de machismo en la sociedad para que predomine la importancia de la igualdad de género. La forma para lograr esta transformación es aumentando la capacitación, divulgación y toma de consciencia de las personas en general, jóvenes y adultos, mujeres y hombres y, sobre todo, las encargadas del diseño de políticas, para que incluyan el objetivo de igualdad de género de forma transversal en todas las políticas públicas. La transformación a favor de la igualdad de género debe realizarse como parte de un trabajo cooperativo de las instituciones y organizaciones, de los sectores público, académico, privado y sociedad civil.

Se recomienda a las instituciones a cargo del cumplimiento de las políticas públicas, en especial de estas 13 últimas aprobadas después del 2016, las revisen y ajusten incluyendo las adaptaciones necesarias para el cumplimiento de cada una de las 9 metas del ODS-5. Un hincapié debe hacerse en redoblar los esfuerzos por lograr el cambio del trato a las mujeres, integrando todas las metas en las diferentes políticas y no sólo en las referidas a género. El esfuerzo de cambio es necesario para desmantelar la institucionalidad tradicional y dominante que discrimina contra las mujeres impidiéndoles su crecimiento humano y el gozo pleno de sus derechos. 
Revista de Política Económica y Desarrollo Sostenible • EISSN: 2215-4167

Vol. 5 (2) • Enero-Junio, 2020: 1-24

Chen Quesada

DOI: https://doi.org/10.15359/peds.5-2.3

Hernández Chaves

URL: http://www.revistas.una.ac.cr/politicaeconomica

Segura Bonilla

La presupuestación y la ejecución de acciones concretas y permanentes a favor del ODS-5 en las instituciones del Estado es tan importante como la aprobación de acuerdos internacionales y de políticas a nivel país. Cada una de las políticas debe tener fuentes claras de recursos cuando sean necesarios, e igualmente indicadores de cumplimiento de los avances logrados.

La prospectiva respecto a la participación de la mujer en las actividades productivas de la sociedad exige mayor consciencia de la igualdad de derechos de estas desde el presente. Debe cambiarse la tradición de los roles de trabajo del hombre y la mujer, especialmente se debe valorar el trabajo doméstico. Es altamente recomendable invertir recursos financieros, logísticos, de tiempo, educación y otros, para lograr la integración de la mujer con todas las metas alcanzadas y que sea aceptada y bien asimilada por toda la sociedad.

Por último, se recomienda continuar investigando sobre las metas del ODS-5 igualdad de género, pero también de cada uno de los 17 Objetivos para el Desarrollo Sostenible. En cada caso tener presente que cada objetivo funciona en la realidad de forma sistémica, vinculado con el resto de los compromisos del desarrollo nacional, o sea que la igualdad de género debe estar presente en forma transversal en todas las políticas de desarrollo de los países.

\section{Referencias}

Aguilar Revelo, L. (2019). Género y cambio climático: retrospectiva y retos. UNED Research Journal, 11(1), S89-S102. https://doi.org/10.22458/urj.v11i1.2326

Arango, M. y Corona, E. (2016). Guía para la igualdad de género en las políticas y prácticas de la formación docente. UNESCO. Recuperado de https://unesdoc.unesco.org/ark:/48223/ pf0000260891

Arias, N., Bustos, A., y Víquez, C. (2016). Transversalización de la Perspectiva de Género: Una mirada crítica a los programas de estudio de la Facultad de Ciencias Sociales. ¿Cuánto hemos avanzado? Universidad En Diálogo: Revista De Extensión, 6(2), 123-144. https://doi. org/10.15359/udre.6-2.7

Benavente, M., y Valdés, A. (2014). Políticas públicas para la igualdad de género Un aporte a la autonomía de las mujeres. Santiago: Chile. Recuperado de https://repositorio.cepal.org/ bitstream/handle/11362/37226/1/S1420372 es.pdf

Centroamérica, P. (2016). Alianza para el Desarrollo Sostenible de Centroamérica. Relaciones Internacionales, 51(2), 153-160. Recuperado de https://www.revistas.una.ac.cr/index.php/ ri/article/view/8871

Chen, E.; Hernández, C.; Muñoz, E. y Segura, O. (2018). Desafíos de la política educativa para el desarrollo sostenible en Costa Rica. Revista Latinoamericana de Educación comparada, 9(14), pp 105-122. 
Revista de Política Económica y Desarrollo Sostenible • EISSN: 2215-4167

Vol. 5 (2) •Enero-Junio, 2020: 1-24

Chen Quesada

DOI: https://doi.org/10.15359/peds.5-2.3

URL: http://www.revistas.una.ac.cr/politicaeconomica

Hernández Chaves

Segura Bonilla

Comisión Interamericana de Derechos Humanos. (2011). Acceso a la información en materia reproductiva desde una perspectiva de derechos humanos. Recuperado de https://www.oas. org/es/cidh/mujeres/docs/pdf/ACCESO\%20INFORMACION\%20MUJERES.pdf

Comisión Nacional de Emergencias. (2016). Política Nacional de Gestión del Riesgo (2016-2030). San José, Costa Rica. Recuperado de https://drive.google.com/file/ d/0ByA2trMWnTn4dS1GOGNlQTFHMU0/view

Consejo Superior de Educación. (2017). Política Educativa: La persona: centro del proceso educativo y sujeto transformador de la sociedad. San José, Costa Rica: MEP. Recuperado de: https://www.mep.go.cr/sites/default/files/page/adjuntos/politicaeducativa.pdf

Consejo Nacional de Planificación Urbana. (2018). Política Nacional de Desarrollo Urbano. San José, Costa Rica: CNPU. Recuperado de https://www.mivah.go.cr/Documentos/politicas directrices planes/PNDU/PNDU Consulta Publica.pdf

Espejel, A., Flores, A., y Castillo, I. (2014). Educación ambiental en el nivel medio superior, desde la perspectiva de género, Tlaxcala, México. Revista Electrónica Educare, 18(3), 17-38. https://doi.org/10.15359/ree.18-3.2

Fernández, D., y Sequeira, P. (2015). Movilidad de mujeres académicas en puestos de decisión: El caso de la Universidad Nacional de Costa Rica. Revista Electrónica Educare, 19(2), 213-230. https://doi.org/10.15359/ree.19-2.13

Health Canada (2003). Exploring Concepts of Gender and Health. Ottawa. Recuperado de: http:// www.hc-sc.gc.ca/english/women/exploringconcepts.htm

Instituto Costarricense de Acueductos y Alcantarillados. (2016). Política Nacional de Agua Potable de Costa Rica 2017-2030. Comisión Interinstitucional. San José, Costa Rica: Instituto Costarricense de Acueductos y Alcantarillados. Recuperado de https://www. aya.go.cr/Noticias/Documents/AyA\%20Pol\%C3\%ADtica\%20Nacional\%20de\%20Agua\%20 Potable\%20de\%20Costa\%20Rica\%202017-2030.pdf

Instituto Costarricense de Acueductos y Alcantarillados. (2016). Política Nacional de Saneamiento en Aguas Residuales 2016-2045. Primera edición. San José, Costa Rica. Recuperado de https://www.aya.go.cr/Noticias/Documents/Politica\%20Nacional\%20 de\%20Saneamiento\%20en\%20Aguas\%20Residuales\%20marzo\%202017.pdf

Instituto Costarricense de Acueductos y Alcantarillados. (2017). Política Nacional de Agua Potable de Costa Rica 2017-2030. San José, Costa Rica. Recuperado de: https://www. aya.go.cr/Noticias/Documents/AyA\%20Pol\%C3\%ADtica\%20Nacional\%20de\%20Agua $\% 20$ Potable\%20de\%20Costa\%20Rica\%202017-2030.pdf 
Revista de Política Económica y Desarrollo Sostenible • EISSN: 2215-4167

Vol. 5 (2) • Enero-Junio, 2020: 1-24

Chen Quesada

DOI: https://doi.org/10.15359/peds.5-2.3

URL: http://www.revistas.una.ac.cr/politicaeconomica

Hernández Chaves

Segura Bonilla

Instituto Nacional de las Mujeres. (2017). Política Nacional para la Atención y la Prevención de la Violencia contra las Mujeres de todas las Edades Costa Rica 2017-2032. 1.a ed. San José: Instituto Nacional de las Mujeres. Recuperado de: https://observatoriodegenero.poderjudicial.go.cr/wp-content/uploads/2018/01/Politica-Nacional-2017-2032.pdf

Instituto Nacional de las Mujeres. (2018). Política Nacional para la Igualdad Efectiva entre Mujeres y Hombres 2018-2030. 1.a ed. San José: Instituto Nacional de las Mujeres. Recuperado de: https://www.inamu.go.cr/web/inamu/pieg2018-2030

Instituto Nacional de las Mujeres. (s. f.). Valorización del trabajo doméstico no remuneradoTDNR. Recuperado de: https://www.inamu.go.cr/valorizacion-del-trabajo-domestico-noremunerado.

Lozano, A., \& Bautista, Q. (2015). Discusiones sobre el género. Apuntes para una reflexión de la violencia de género en la universidad. Revista ABRA, 35(51), 1-19. https://doi.org/10.15359/abra.35-51.8

Melero, N., \& Limón, D. (2017). Educar en espacios comunitarios de participación, cooperación y ecodesarrollo desde una perspectiva ecofeminista. Revista Electrónica Educare, 21(1), https://doi.org/10.15359/ree.21-1.22

MIDEPLAN. (2016). Guía para la elaboración de políticas públicas. MIDEPLAN. San José, Costa Rica. Recuperado de https://www.inder.go.cr/acerca del inder/politicas publicas/ documentos/Guia-de-Elaboracion-de-PP.pdf

Ministerio de Ambiente y Energía. (2018). Política de Adaptación al Cambio Climático 2018-2030. San José, Costa Rica. Recuperado de http://www.pgrweb.go.cr/DocsDescargar/Normas/ No\%20DE41091/Version1/Politica ADAPTACION 24 abril.pdf

Ministerio de Ambiente y Energía. (2018). Política Nacional de Producción y Consumo Sostenible (2018-2030). San José, Costa Rica. Recuperado de http://www.digeca.go.cr/sites/ default/files/documentos/propuesta de politica nacional de consumo y produccion sostenibles 15-12-2017.pdf

Ministerio de Ambiente y Energía. (2018). Política Nacional de Humedales. San José, Costa Rica. Recuperado de: https://presidencia.go.cr/wp-content/uploads/2017/03/Politica-Nacionalde-Humedales-1.pdf

Ministerio de Ambiente y Desarrollo Sostenible. (2017). Política Nacional de Cambio Climático. Colombia, Bogotá. Recuperado de http://www.minambiente.gov.co/images/ cambioclimatico/pdf/Politica Nacional de Cambio Climatico PNCC /PNCC Politicas Publicas LIBRO Final Web 01.pdf

Ministerio de Asuntos Exteriores y de Cooperación. (2017). Política de Responsabilidad Social. San José, Costa Rica. Recuperado de: https://www.aedcr.com/sites/default/files/pn responsasocialcr 2017.pdf 
Revista de Política Económica y Desarrollo Sostenible • EISSN: 2215-4167

Vol. 5 (2) •Enero-Junio, 2020: 1-24

Chen Quesada

DOI: https://doi.org/10.15359/peds.5-2.3

Hernández Chaves

URL: http://www.revistas.una.ac.cr/politicaeconomica

Segura Bonilla

Ministerio de Desarrollo Humano e Inclusión Social. (2016). Política Nacional para la Atención Integral a las Personas en Situación de Abandono y Situación de Calle, 2016-2026. I Edición. Gobierno de la República de Costa Rica. Consejo Presidencial Social. Recuperado de https://presidencia.go.cr/wp-content/uploads/2016/06/Pol\%C3\%ADtica-Integral-paraPersonas-en-Situaci\%C3\%B3n-de-Abandono-y-Calle.pdf

Ministerio de Economía y Finanzas-Consejo Nacional de Competitividad y Formalización. (2019). Política Nacional de Competitividad y Productividad. Perú Recuperado de https://www. mef.gob.pe/contenidos/archivos-descarga/Politica_Nacional de Competitividad y Productividad.pdf

Naciones Unidas. (2018). La Agenda 2030 y los Objetivos de Desarrollo Sostenible: una oportunidad para América Latina y el Caribe (LC/G.2681-P/Rev.3), Santiago.

Orjuela, A. (2012). El concepto de violencia de género en el Derecho Internacional de los Derechos Humanos. Revista Latinoamericana de Derechos Humanos, 23(1), 89-114. Recuperado de https://www.revistas.una.ac.cr/index.php/derechoshumanos/article/view/5290

Pichardo, A. (1993). Planificación y programación social. HV Manitas, Buenos Aires, Argentina.

Poder Judicial de Costa Rica, Observatorio de Violencia de Género contra las Mujeres y Acceso a la Justicia. (2019). Recuperado de https://observatoriodegenero.poder-judicial.go.cr/soyespecialista-y-busco/estadisticas/femicidio/

Programa Estado de la Nación. (2019). Informe Estado de la Nación. 25a Ed. Servicios Gráficos AC. San José, Costa Rica. Recuperado de http://repositorio.conare.ac.cr/ handle/20.500.12337/7808

Rosales, A., \& Salinas, F. (2017). Educación sexual y género en primarias mexicanas ¿qué dicen los libros de texto y el profesorado? Revista Electrónica Educare, 21(2), 1-21. https://doi. org/10.15359/ree.21-2.11

Scott, P. (1990) Introducción a la investigación y evaluación educativa. Universidad de San Carlos, USAC. Llerena, S.A. Guatemala. Disponible en: https://es.unesco.org/creativity/sites/ creativity/files/iucd manual metodologico 1.pdf

UNESCO. (2014). Indicadores UNESCO de cultura para el desarrollo: Manual Metodológico. Organización de la Naciones Unidas para la Educación, la Ciencia y la Cultura. Place de Fontenoy, 75352, París, Francia.

\section{Notas al final}

1 Catedrática de la Universidad Nacional, en la División de Educación para el Trabajo (CIDE-DET). Máster en Administración Educativa de New Mexico University, USA. Ex-Directora del Consejo Nacional de Enseñanza Superior Universitaria Privada (CONESUP) de Costa Rica. Investigadora y docente en el Centro de Investigación y Enseñanza (CIDE) y actual Coordinadora de la Maestría en Gestión Educativa con énfasis en Liderazgo, Universidad Nacional de Costa Rica. 
Revista de Política Económica y Desarrollo Sostenible • EISSN: 2215-4167

Vol. 5 (2) • Enero-Junio, 2020: 1-24

DOI: https://doi.org/10.15359/peds.5-2.3

Chen Quesada

URL: http://www.revistas.una.ac.cr/politicaeconomica

Hernández Chaves

Segura Bonilla

2 Máster en Planificación y Administración Curricular de la Universidad Latinoamericana de Ciencia y Tecnología (ULACIT), Costa Rica. Especialidad en Investigación Interdisciplinaria de la UNAM, México. Ex Asesora Nacional de Inglés en la Dirección de Desarrollo Curricular del Ministerio de Educación Pública. Investigadora y docente de inglés en la Facultad de Ciencias Sociales de la Universidad Nacional de Costa Rica, carolina.hernandez.chaves@una.cr

3 Economista con Maestría de la Universidad de Londres, Queen Mary and Westfield College, Inglaterra y Ph.D. en Economía de la Innovación y el Cambio Tecnológico de Aalborg University, Dinamarca. Ex-Presidente del Instituto Nacional de Aprendizaje, ex-Rector de la Universidad Nacional, Costa Rica. Investigador y docente en el Centro Internacional de Política Económica para el Desarrollo Sostenible (CINPE-UNA). olman.segura.bonilla@una.ac.cr 